\title{
From ecology to genetics and back: the tale of two flounder species in the Baltic Sea
}

\author{
Jokinen, Henri Matias
}

2019

Jokinen , H M , Momigliano , P \& Merilä , J 2019 , ' From ecology to genetics and back: the tale of two flounder species in the Baltic Sea ' , ICES Journal of Marine Science , vol. 76 , pÿno. 7 , pp. 22672275 . https://doi.org/10.1093/icesjms/fsz151

http://hdl.handle.net/10138/310520

https://doi.org/10.1093/icesjms/fsz151

cc_by

acceptedVersion

Downloaded from Helda, University of Helsinki institutional repository.

This is an electronic reprint of the original article.

This reprint may differ from the original in pagination and typographic detail.

Please cite the original version. 
This is the AUTHORS' ACCEPTED MANUSCRIPT, for the published article, please see: https://academic.oup.com/icesjms/article-abstract/76/7/2267/5552826?redirectedFrom=fulltext

Full citation:

Jokinen H., Momigliano P. \& Merilä J., 2019. From ecology to genetics and back: the tale of two flounder species in the Baltic Sea. ICES Journal of Marine Science 76, 2267-2275.

From ecology to genetics and back: the tale of two flounder species in the Baltic Sea

Henri Jokinen $^{b^{*}}$, Paolo Momigliano ${ }^{a} \& J_{\text {Jha Meriläa }}$

${ }^{\mathrm{a}}$ Ecological Genetics Research Unit, Research Program in Organismal and Evolutionary Biology, University of Helsinki PO Box 65, FI-00014, Finland

${ }^{\mathrm{b}}$ Tvärminne Zoological Station, University of Helsinki, J.A. Palménin tie 260, FI-10900 Hanko, Finland

*Corresponding author: tel: +358407721763; e-mail: henri.jokinen@helsinki.fi. 


\begin{abstract}
Recent years have brought the realization that evolutionary changes, including speciation, driven by selection can occur in ecological time scales. However, recent evolutionary events can be hard to detect and may easily go unnoticed. For harvested species, such cryptic diversity, may lead to suboptimal resource management. These points are illustrated by cryptic ecological speciation in Baltic Sea flounders. Although early ecological studies identified two ecotypes of European flounder (Platichthys flesus) based on spawning of pelagic and demersal eggs, genomic studies only recently demonstrated that they were reproductively isolated species, $P$. flesus and $P$. solemdali, which underwent ecologically driven speciation following the end of the last glaciation. These morphologically indistinguishable species are harvested within a mixed-stock fishery. Landings from this fishery in many areas of northern Baltic Sea have declined since the mid-1980s, and the drop in the Gulf of Finland (GoF) catches has been particularly dramatic ( $90 \%)$. Genetic analyses of historical otolith samples from GoF catches have revealed that back in 1983 the fishery unknowingly targeted primarily $P$. flesus, whereas thereafter almost exclusively $P$. solemdali. Hence, the case of two flounder species illustrates $(i)$ how ecological studies stimulated genetic investigations leading to discovery of ecological speciation, and (ii) how cryptic species turnover discovered with genetic tools in turn improved ecological understanding of flounders with benefits to management and conservation.
\end{abstract}

Keywords: ecological speciation, cryptic species, genomics, mixed-stock fishery, otolith, Platichthys flesus, Platichthys solemdali 


\section{Introduction}

While developments in the fields of ecology and genetics have largely proceeded independently of each other, ecology and genetics have a long history of interaction (see reviews in Berry et al., 1992). Ecology is inextricable from genetics, as both neutral and adaptive changes in organisms' genomes are shaped by biotic and abiotic environmental factors. For decades, population geneticists have studied how landscape physiognomy, environmental features and inter-specific interactions shape neutral and adaptive genetic diversity within and among populations (Manel et al., 2003). Similarly, genetic tools have become essential in the study of biodiversity: genetic approaches are needed for correct species/ecotype identification and to quantify biodiversity, particularly in taxonomic groups were cryptic species are common (Bickford et al., 2007).

The potential for fruitful interactions between ecology and genetics is particularly evident in the study of marine ecosystems. As argued by Knowlton (2000), cryptic species/populations may be more common in the marine environment, because a) direct observation of animal behaviour is more challenging, and b) mate choice and gamete recognition rely on unobservable chemical cues. Marine organisms also represent potentially excellent model systems for ecological geneticists as marine populations are usually characterized by large effective population sizes and high degree of habitat connectivity (and hence potentially high levels of gene flow). Large effective population sizes should facilitate evolutionary responses to selection, as more genetic variation is produced via random mutation and potentially adaptive alleles are less likely to be stochastically lost (Allendorf et al., 2010; Savolainen et al., 2013). Similarly, high levels of gene flow provide an effective means for the spread of potentially beneficial mutations (Schluter and Conte, 2009).

Nevertheless, the study of wild marine populations with the tools of population genetics has been challenging. Detecting genetic structure in large, open populations is a difficult task (Cano et al., 
2008; Nielsen et al., 2009). Similarly, as the rate of decay of linkage disequilibrium around genomic regions under selection is a positive function of $N_{\mathrm{e}}$ (Hill and Robertson, 1968) detection of adaptive population differentiation with a limited number of genetic markers is difficult as genomic regions affected by linked selection may be small (Cano et al., 2008; Nielsen et al., 2009), and genomic resources for non-model marine organisms are still limited. Population genetic studies based on limited number of genetic markers can therefore easily overlook existing genetic differentiation not only among particular populations or stocks (e.g. Lamichhaney et al., 2012; Hemmer-Hansen et al., 2013), but also among recently diverged and reproductively isolated biological species (Momigliano et al., 2017). Consequently, the power of population genetic studies to inform ecologists-and vice versa-has been limited in the past due to analytical constraints, but the situation has been rapidly changing during the past decade.

Recent and rapid advances in sequencing technologies and downstream bioinformatics tools provide geneticists with means to overcome-or at least alleviate-some of these challenges. For instance, recent studies using large set of markers and whole genome data have discovered that populations of many marine fish species are more structured than previously anticipated (e.g. Lamichhaney et al., 2012; Guo et al., 2015; Berg et al., 2016), and this structuring often corresponds to discontinuities in physiochemical and ecological conditions in the sea (e.g. Lamichhaney et al., 2012; Selkoe et al., 2016). Genomic data have allowed identification of candidate loci underpinning local adaptations in various ecological conditions (Lamichhaney et al., 2012; Berg et al., 2016; Momigliano et al., 2017), and this new information on adaptive genetic variation can be integrated in the delineation of conservation and management units to protect biodiversity (Funk et al., 2012; Lamichhaney et al., 2012; Berg et al., 2016). 
The results from these studies have started to inform managers. For instance, candidate gene (Fevolden and Pogson, 1997) and genomic (Hemmer-Hansen et al., 2013) studies led to the identification of distinct ecotypes of Atlantic cod (Gadus morhua) co-occurring in the large Norwegian Atlantic cod fishery consisting of North East Arctic cod (NEAC) and Norwegian Coastal cod (NCC). The two ecotypes can be identified by different alleles in the Pantophysin gene, which is located within one of the chromosomal inversions associated with different spawning and migratory behaviours (Berg et al., 2016). The NEAC is abundant and stable while the NCC stock has undergone severe declines. By measuring in real time the composition of the mixed stock, the Norwegian Directorate of Fisheries allows exploitation of the abundant NEAC stock while simultaneously protecting the fragile NCC stock by opening and closing fisheries depending on the relative proportion of each (Dahle et al., 2018).

Increasing amounts of genetic evidence have uncovered overlooked ecological diversity. In the same way, ecology has informed genetic studies about previously unidentified speciations (Hyde et al., 2008; Momigliano et al., 2017: Fig 1) and has even led to discoveries of turnovers in the abundance of cryptic species and ecotypes (Bonanomi et al., 2015; Dahle et al., 2018; Momigliano et al., 2019). The recent discovery of a Baltic Sea flounder species pair (Momigliano et al., 2017) is a case in point: insights from ecology (Solemdal, 1967; Solemdal, 1973; Nissling et al., 2002) spurred genetic investigations (Hemmer-Hansen et al., 2007; Florin and Höglund, 2008; Momigliano et al., 2017; Momigliano et al., 2018), whose results in turn provided ecological information relevant for resource management and conservation (Momigliano et al., 2019).

Flounders (Platichthys spp.) are some of the relatively few marine species that live in the low salinities (bottom salinity 0-20 psu; Fig. 1A) of the Baltic Sea, a large brackish water basin that was formed in connection to the North Sea at the end of the last glaciation, $~ 8.5$ kya (Gustafsson and 
Westman, 2002). While the other four regularly occurring species of flatfishes (Pleuronectiformes) in the Baltic Sea are found most frequently in the south, flounders are the most common and widely distributed flatfishes throughout the sea, except in the northern- and eastern-most parts (i.e. ICES SD 31, northern part of SD 30 and eastern-most part of SD 32; Fig. 1B) where nearly freshwater conditions prevail (ICES, 2017).

From the descriptive studies of early naturalists (e.g. Hensen, 1884), through experimental investigations of reproductive characteristics (Solemdal, 1967; Solemdal, 1973; Nissling et al., 2002; Nissling and Dahlman, 2010), to population genetic studies (Hemmer-Hansen et al., 2007; Florin and Höglund, 2008), observations and investigations on Baltic Sea flounders have led to the recognition of two distinct but closely related species with differences in several traits (Momigliano et al., 2017; Momigliano et al., 2018). Importantly, the two flounder species in the Baltic Sea differ in reproductive behaviour. The European flounder $P$. flesus spawns pelagic eggs in deep offshore basins of the southern and central Baltic Sea where salinity is high enough ( $>10.7 \mathrm{psu}$, see Fig. 1A) for eggs to achieve neutral buoyancy (Nissling et al., 2002). The newly described Baltic flounder $P$. solemdali (Momigliano et al., 2018) spawns demersal eggs in shallow coastal waters and banks and can reproduce successfully in salinities as low as 6 psu (Momigliano et al., 2018). The species are considered parapatric, co-occurring in some areas of the central Baltic Sea (Momigliano et al., 2017; Momigliano et al., 2018). However, the distinct reproductive strategies associated with differences in sea water salinity result in spatial segregation during spawning (Solemdal, 1967; Nissling et al., 2002; Nissling and Larsson, 2018).

The aim of this paper is to review the case of the two Baltic Sea flounders in the light of recent findings, as well as to re-analyse some of published data to further illuminate the challenges (and prospects) associated with genetic work in marine organisms. We first review how early ecological 
studies on reproductive biology laid the basis for the genetic work that uncovered the rapid speciation of the Baltic Sea flounders through selection of traits causing reproductive isolation. Second, we show how ecological and genetic studies played a role in revealing this cryptic speciation in which differentiation is confined to ecologically important reproductive traits that are difficult to observe. Third, we consider the role of large $N_{e}$ in producing low levels of differentiation at neutral markers, and how this can-depending on which kind of genetic methods are used-either conceal or facilitate discovery of small but biologically significant genetic differentiation. Finally, we review how genetic species identification based on markers under selection revealed that Baltic Sea flounders represented a mixed-species fishery, and also lead to detection of dramatic temporal turnover in species composition.

\section{From ecology to genetics-discovery of cryptic ecological speciation}

Understanding the processes by which new species arise-"the mystery of mysteries" (Darwin, 1859)-has been a major challenge facing evolutionary biologists. The idea that adaptation by natural selection has played a central role in the multiplication of species over time goes as far back as Darwin, who suggested 'varieties' will acquire with time more modifications and become different species (Darwin, 1859). However, it was not until the second half of the 1990s that ecology was brought to the forefront of speciation research (Schluter and Rambaut, 1996). Since then, there has been a surge of research on ecological speciation, the process by which ecological selection generates reproductive isolation as a by-product (Nosil, 2012).

While there is a plethora of examples of ecological speciation in terrestrial and freshwater environments (Nosil, 2012), evidence for ecological speciation in marine fish is still relatively scarce (Puebla, 2009) with few cases where ecological factors have been indirectly implicated in speciation events in the sea (e.g. Rocha et al., 2005; Hyde et al., 2008). This is surprising: barriers 
to dispersal are rarely absolute in the sea, hence models of speciation that can operate in the presence of gene flow, such as ecological speciation, are likely to be important in explaining marine biodiversity (Puebla, 2009). The flounder species-pair provides therefore a useful model to study evolution driven by ecological selection in marine systems, and in particular to investigate the mechanisms of speciation in the absence of absolute barriers to gene flow.

Despite having a parapatric distribution, $P$. flesus and $P$. solemdali in the Baltic show strongly bimodal genotypic clustering based on genome-wide SNP markers and strong reproductive isolation (Momigliano et al., 2017). Divergence across the genome is weak, but genomic signatures show evidence of selection associated with reproductive traits that are under ecological selection (Momigliano et al., 2017), a pattern suggestive of a recent ecological speciation event.

Daverat et al. (2012) suggested that $P$. flesus could be adapted to spawn in freshwater as well as brackish and marine environments in estuaries of France and Spain. It seems therefore likely that the genomic architecture underlying the adaptations necessary to invade the Baltic Sea may pre-date the end of the last glaciation. If such brackish/freshwater adapted alleles were present in the North Sea marine population, they would provide the raw material on which selection could act upon during the invasion of the Baltic Sea. Momigliano et al. (2017) postulated that P. solemdali and the Baltic Sea population of the P. flesus originated via two independent invasions into the Baltic Sea (Fig. 1D) from the same ancestral population. According to their reconstruction, the invasion that gave rise to P. solemdali occurred shortly after the connection of the Baltic Sea to the North Sea, $\sim 7.2$ thousand years ago (kya). At this time, salinity in the entire Baltic Sea was below the threshold ( $\sim 10 \mathrm{psu})$ at which spawning of pelagic eggs is possible in extant marine species inhabiting this area (Nissling et al., 2002; Nissling and Larsson, 2018; Fig. 1C). It was inferred that ecological selection to very low salinities led to the evolution of a demersal spawning strategy (Momigliano et 
al., 2017). In fact, $P$. solemdali shows unique adaptations in egg morphology and sperm physiology which enable demersal spawning in low salinity conditions (Solemdal, 1967; Lønning and Solemdal, 1972; Solemdal, 1973; Nissling et al., 2002). In contrast, the invasion that gave rise to the Baltic population of the pelagic spawning $P$. flesus is estimated to have occurred $\sim 5$ kya, at which time salinity in the entire Baltic Sea was above $10 \mathrm{psu}$, and pelagic spawning could have occurred in most of the Baltic Sea (Fig. 1C). This invasion also faced selection to lower salinity than usually experienced by the ancestral population, but still retained the pelagic strategy as it was physically possible. Demographic modelling revealed that introgression between the two Baltic Sea flounders is limited, suggesting that reproductive isolation had been established within $\sim 1000$ generations of initial allopatry (Momigliano et al., 2017).

One hypothesis is that ecological selection on egg specific gravity, reproductive behaviour and sperm physiology led to reproductive isolation. P. flesus spawning in the Baltic Sea is restricted to deep-water basins where salinity is sufficiently high for eggs to achieve neutral buoyancy below the $11 \mathrm{psu}$ isohaline. Similar to other marine fishes adapted to the low salinity waters of the Baltic (e.g. Kijewska et al., 2016), P. flesus shows adaptations in egg density. Eggs produced in the Baltic Sea are larger and with thinner chorions and have lower specific gravities than those in the North Sea, enabling them to achieve neutral buoyancy in low-salinity waters (Lønning and Solemdal, 1972; Nissling et al., 2002). Eggs of P. solemdali differ in morphology: they are slightly larger and with lower specific gravity than North Sea $P$. flesus, but smaller with thicker chorions than those of Baltic Sea P. flesus (Lønning and Solemdal, 1972); adaptations which enabled P. solemdali to spawn demersally in the coastal waters of the Baltic Sea (Nissling et al., 2002). Differences in egg morphologies preclude the possibility of each species effectively spawning in the reproductive habitat of the other, providing a link between ecological selection and reproductive isolation. Selection on sperm motility may also be an important factor in the establishment of reproductive 
isolation between the two species; the activation of sperm from $P$. flesus requires salinities of above 10 psu vs. 3 psu for sperm from demersal flounders (Nissling et al., 2002). Thus, P. flesus sperm activates at salinities well above the levels typically encountered in P. solemdali spawning sites (Solemdal, 1973; Nissling et al., 2002; Momigliano et al., 2018).

How these species remained reproductively isolated in secondary contact (Momigliano et al., 2017) remains puzzling. Gene flow between populations and recombination can break apart adaptive combinations of alleles, resulting in reduced fitness (Ortiz-Barrientos et al., 2016). For this reason, reproductive isolation between parapatric populations is expected to be facilitated by mechanisms reducing recombination, especially among alleles reducing the incidence of hybridization (Butlin, 2005; Ortiz-Barrientos et al., 2016).

Momigliano et al. $(2017 ; 2018)$ discovered that the two species are nearly fixed for alternative alleles of two outlier loci, locus 886_19 and Locus 3359_4. Here we further explore these data by aligning DNA sequences from Momigliano et al. (2017) to a draft genome of P. solemdali (Momigliano and Merilä, unpublished data) and estimate the amount genetic differentiation with $F_{\text {ST }}$ along the two scaffolds where these two outlier genes are located (Fig. 2). We then mapped to the same scaffolds the cDNA of the closest BLAST matches of the Lpar3, VtgA and DNAH5 genes in Japanese flounder Paralicthys olivaceus and barfin flounder Verasper moseri. Locus 3559 lies within the coding region of the Dynein Axonemal Heavy Chain 5 (DNAH5) gene, where alternative alleles produce an amino-acid change (Momigliano et al., 2017). Dyneins are proteins playing a central role in the beating of flagella, and therefore in sperm (Turner, 2003). Deleterious mutations within DNAH5 are associated with spermatozoa dysfuntion (Zuccarello et al., 2008). The SNP $886 \_19$ is also nearly fixed for alternative alleles in Baltic Sea populations of $P$. flesus and in $P$. solemdali. Interestingly, this SNP is polymorphic in North Sea populations where both the alleles 
characteristic of $P$. solemdali and the Baltic population of $P$. flesus are common. Momigliano et al. (2017) identified a gene close to that locus, the Lysophosphatidic acid receptor 3 (lpar3).

We also identified the Vitellogenin A gene adjacent to the outlier (VgA; Fig. 2B). Vitellogenins are precursor proteins of egg yolk, and in $V$. moseri, the relative proportion of the proteins produced by the $\mathrm{VgA}$ and $\mathrm{VgB}$ genes determines the buoyancy of pelagic eggs (Matsubara et al., 1999), the same trait that could have acted as a magic trait (i.e. a trait under selection that directly causes reproductive isolation; sensu Servedio et al., 2011) in P. flesus-P. solemdali speciation. Lpar 3 is also involved in reproduction: it codes for Lysophosphatidic acid (LPA), a cell membrane phospholipid metabolite that functions as an extracellular signal (Ye et al., 2010). LPA plays an important role in spermatogenesis, ovarian function, fertilization and embryo early development (Ye et al., 2010).

These outlier genes are promising candidates for "magic traits" (Servedio et al., 2011). However, to link the observed patterns of heterogeneous genomic differentiation, the ecological selective pressures and the rise of reproductive isolation, we need to combine more genetic data with reciprocal acclimation experiments aimed at identifying the roles of these candidate genes in ecological divergence. Although the genetic studies of flounders founded on initial findings from ecology have uncovered a speciation event, several questions about the drivers and mechanisms of this speciation remain to be tested. Future genetic studies are needed also to screen flounder genomes for additional candidate genes that may have been involved in speciation. The number of markers used in Momigliano et al. (2017) was modest (2051 SNPs) relative to size of the flounder genome $(0.53 \mathrm{~Gb}$, about $1 \mathrm{SNP}$ every $50 \mathrm{~kb})$, and many highly differentiated candidate genes may have been missed as linkage disequilibrium (LD) in flounders-as well as in other marine fish species with large $N_{e}$, see Hemmer-Hansen et al. (2014) -decays rapidly (Fig. 3). Whole genome 
sequencing may reveal more candidate genes. If the decay of LD is rapid as fast as shown for flounders (Fig. 3) and in other species (Hemmer-Hansen et al., 2014), it is likely that earlier genome scans of marine fishes based on low marker numbers may have overlooked highly divergent genomic regions of ecological significance.

\section{From genetics to ecology-hidden biodiversity, population biology and community dynamics}

For ecologist, information on growth, maturation, fecundity, and on survival and reproductive rates are essential to understand the dynamics and ecologies of populations. These parameters can be accurately inferred only if they are estimated from single demographically independent population. However, when closely related and morphologically similar species co-occur in the same geographic area, obtaining accurate species-specific estimates may be challenging. Selection on non-visual reproductive traits, such as chemical mating signals, gamete physiology and spawning behaviour, may result in ecologically divergent, but morphologically cryptic species (Bickford et al., 2007). Similarly, adaptation to similar extreme environmental conditions can promote morphological stasis (Bickford et al., 2007), hindering morphological differentiation between sibling species. This has likely been the case with the flounder species-pair in the Baltic Sea. The rapid speciation of the younger Baltic flounder from an ancestral European flounder may have been too recent for morphological differences of less selective importance to coevolve. Moreover, overlapping habitats and shared ecological features typical for flatfishes, including a demersal life after settling, similar feeding behaviour and common predators (Gibson et al., 2015) may produce only weak divergent selection on adult morphology. While reproductive characteristics such as egg morphology and sperm physiology are important diagnostic traits, their utility in identifying individuals of each species in the wild is limited, as they can be observed only in spawning individuals. Thus, the most practical way to identify the two Baltic Sea flounders is with diagnostic genetic markers, such as SNPs. 
There has been growing interest in characterizing biological traits in flounders at the Baltic wide scale, such as migration (Aro and Sjöblom, 1983), fecundity (Nissling and Dahlman, 2010) and body size (Erlandsson et al., 2017). However, the samples used in these studies likely included mixtures of the two species and the results may not be reliable. Reproductive characteristics, such as egg specific gravity, sperm activation and motility, fecundity and sperm production can differ in the two species (Nissling et al., 2002; Nissling and Dahlman, 2010; Nissling et al., 2017; Nissling and Larsson, 2018) and hence, may lead to different projections of, for instance, population growth depending on the composition of the samples over space and time. Hence, accurate estimates of species-specific traits aided by genetic species identification should improve the inference from population ecological studies of flounders.

Environmental factors such as temperature and salinity are not stable in time, and even subtle changes can affect the distributions of both flounder species in the Baltic Sea, where they live close to their physiological limits. Decreasing salinity (caused by climatechange: Vuorinen et al., 2015) and the ongoing deoxygenation of the Baltic Sea as (a result of eutrophication: Carstensen et al., 2014) will likely result in range contractions of the two flounders among many other marine species in the Baltic Sea (Vuorinen et al., 2015). Diagnostic tools for species identification provided by genetics are essential to accurately monitor species-specific responses, and to put management and conservation on sound scientific footing.

The identification of cryptic species may also explain past population and community dynamics, which may be masked due to inaccurate estimates of species composition. Again, the two Baltic Sea flounders provide an excellent example. Flounder landings and Catch per Unit Effort in the Gulf of Finland have declined by nearly $90 \%$ in the past four decades (Jokinen et al., 2015; ICES, 2017). 
The trend was long assumed to reflect a gradual decline of the local demersal population (now $P$. solemdali), but recent work indicates that the decline reflects chiefly the cessation of $P$. flesus larval subsidies from the south. This led to the near disappearance of the previously dominant $P$. flesus, and was succeeded by a decline of resident P. solemdali (Momigliano et al., 2019). This cryptic turnover was likely due to the degradation of environmental conditions-i.e., combination of decreased deep water salinity and oxygen content-for reproduction of $P$. flesus in faraway spawning grounds (Fig. 1C). Subsequent local environmental degradation-i.e., decreased salinity and habitat degradation due to eutrophication-may then have affected the reproductive success of P. solemdali and other living conditions of both species. This exemplifies how cryptic community components respond to various environmental factors at local and regional scales, creating a complex ecological picture seen only with genetic data. Having this picture clarified is central for making sensible study designs for questions intimately related to the composition and dynamics of these species and for the interpretation of the results.

\section{Management implications and considerations}

The Baltic Sea flounder species pair provides an example of what was previously considered to be a single-species fishery, but is in reality a mixed-stock fishery targeting two distinct species the relative proportion of which have changed through time due to shifts in environmental conditions (Momigliano et al., 2019).

Hidden stock structure within mixed-stocks fisheries might hinder appropriate management, as stock components with distinctive biological and ecological characteristics are managed with assessments using parametrization of only the dominating stock component, or some mixture that represents neither stock (Hauser and Carvalho, 2008; Reiss et al., 2009). Spatiotemporal shifts in the relative contribution of stock components, as each can respond differently to fishing pressure 
and environmental change, is likely to go unnoticed (Bonanomi et al., 2015). A failure to recognize such stock diversity can lead to under- or over-harvest of the separate components of the fishery ( Hauser and Carvalho, 2008; Hutchinson, 2008) and may ultimately lead to a fishery collapse, as in the West Greenland cod fishery (Bonanomi et al., 2015).

Local flounder populations in the Baltic Sea are currently managed as single stocks of the $P$. flesus ecotypes (ICES, 2017). This despite the recent discoveries by Momigliano et al. (2017), recognizing two instead of one species, and revealing mixed local assemblages with varying proportions of both species. Momigliano et al. (2019) screened diagnostic SNPs in DNA extracted from archival otolith samples to reconstruct the species-composition of flounder assemblage over the past four decades. In the early 1980s, P. flesus dominated the flounder assemblage, disappeared by the early 1990s, and returned by the early 2000s remaining in low proportions thereafter (Fig. 4). The disappearance of $P$. flesus coincided with worsening environmental conditions in its closest spawning ground in the preceding 4-6 years (Momigliano et al., 2019) and closely mirrored the temporal drop in flounder landings (ICES, 2017): fishery-independent surveys indicated a decline in the mixed flounder stock (Jokinen et al., 2015). These results together support the notion that Northern Baltic Sea $P$. flesus constitute a sink population sourced by recruits from the southern stock. Such sourcing would occur under conditions favourable for spawning of the southern stock, which are characterized by sufficient salinity $(>11 \mathrm{psu})$ and oxygen concentrations $(>1 \mathrm{ml} / \mathrm{L})$ during spawning time (Nissling et al., 2002; Nissling et al., 2017).

The hidden temporal dynamics of the two flounders resemble the case of the West Greenland cod fishery, where a fishery collapse was caused by the successive disappearance of previously unobserved, demographically independent stock components as a response to deteriorating environmental conditions and overfishing (Bonanomi et al., 2015). A serious consequence of 
managing a mixed stock as a single stock fishery is unintentional overexploitation of the weaker stock component (Hutchinson, 2008), although differences in ecologically important traits within the stock might potentially increase the overall resilience to exploitation and disturbance (Hauser and Carvalho, 2008). Also, as different stock components with specific characteristics can thrive during different environmental conditions and climatic regimes, having knowledge of such biocomplexity in a fish stock is critical for understanding the responses to environmental change and the implication for management and conservation (Hilborn et al., 2003). The tale of two flounders thus highlights the importance of recognizing cryptic genetic diversity and studying temporal heterogeneity, and the underlying causes, in the composition of mixed-stock fisheries. This is important for development of adaptive management of marine resources in the face of anthropogenic changes. Without access to genetic tools enabling species identification, one of the two flounder species would have almost completely disappeared from a local fishery before scientists and managers noticed its presence. In fact, genetic data are, in many cases, the most important evidence for identifying independent stocks and management units (Hauser and Carvalho, 2008; Reiss et al., 2009). However, their incorporation into fisheries models is still not done routinely everywhere as genetic tools for rapid identification of stock components are not available for most species, and therefore, widespread integration of genetics into fishery policy has been slow for many species (Reiss et al., 2009).

Unambiguous assignment of individuals to the right stock component would enable more accurate estimates of taxon- or population-specific growth rates and fecundity that might exhibit considerable divergence between stocks and be instrumental for understanding ecological responses to environmental change and exploitation. Thus, this capability aids policing fishery regulations and managing fisheries (Reiss et al., 2009). The genetic test developed and refined by Momigliano et al. $(2018$; 2019) provides a means for the unambiguous identification of Baltic Sea flounder species 
and could be employed for real-time monitoring and mixed-stock analyses of catches. Genetic management tools have successfully been implemented in the mixed-stock cod fishery along the Norwegian coast to limit exploitation of less abundant stock components (Dahle et al., 2018). These tools could create the bases for the effective adaptive management of $P$. flesus and $P$. solemdali in the Baltic Sea by enabling the estimation of demographic changes as well as responses to management for each species separately. Stock components of Baltic Sea flounder could be assessed yearly in areas where the two species are known to markedly overlap. The sampling could be conducted on regular basis, perhaps weekly, during the main fishing season of flounders. Tissue samples from sufficient number of fishes could be collected by authorities from the catches by larger vessels and from contract fishermen in small-scale coastal fisheries. Based on the assessment of the species' composition of catch and on stock assessments depending on estimated stock status for each species, the mixed flounder fishery could be regulated to limit or promote fishing of either of the species, according to the situation. Real-time monitoring would enable real-time management in such cases in which one of the mixed-stock species is less abundant.

\section{Conclusions and concluding remarks}

We opened this article by pointing out that when viewed from an evolutionary perspective, genetics and ecology have always been in an intimate relationship as few evolutionary inferences would be complete and satisfactory without understanding both genetic mechanisms and ecological drivers of evolutionary changes. Using the species pair of Baltic Sea flounders as an example, we have shown how early ecological studies made observations which lead to genetic investigations and the discovery of the separate species. This discovery and genetic tools adopted from that study provided means to uncover a rapid and cryptic community composition turnover that had taken place in a decadal time scale. Hence, the tale of the two flounders illustrates how ecological studies motivated genetic investigations, and how the results of these studies fed back into novel insights on the 
ecologies of these two species. Apart from the contributions to the fundamental science, one of the outcomes of this synergism concerns the management of marine resources in the face to rapid anthropogenic change.

\section{Acknowledgements}

Our research was supported by grants from the Academy of Finland (JM: 129662, 134728 and 218343; PM: 316294) and Helsinki Institute of Life Science (HiLIFE; JM: 797011029).

\section{Author contributions}

All authors contributed equally to this paper. 


\section{References}

Allendorf, F. W., Hohenlohe, P. A., and Luikart, G. 2010. Genomics and the future of conservation genetics. Nature Reviews Genetics, 11: 697.

Aro, E., and Sjöblom, V. 1983. The migration of flounder in the northern Baltic Sea. International Council for the Exploration of the Sea C.M.1983/J: 26.

Bendtsen, J., Söderkvist, J., Dahl, K., Hansen, J. L., and Reker, J. 2007. Model simulations of blue corridors in the Baltic Sea. BALANCE interim Report: 26.

Berg, P. R., Star, B., Pampoulie, C., Sodeland, M., Barth, J. M., Knutsen, H., Jakobsen, K. S., et al. 2016. Three chromosomal rearrangements promote genomic divergence between migratory and stationary ecotypes of Atlantic cod. Scientific Reports, 6: 23246.

Berry, R., Crawford, T., and Hewitt, G. 1992. Genes in Ecology, Blackwell Scientific Publishing, Oxford and Bostin (Massachussetts). 534 pp.

Bickford, D., Lohman, D. J., Sodhi, N. S., Ng, P. K. L., Meier, R., Winker, K., Ingram, K. K., et al. 2007. Cryptic species as a window on diversity and conservation. Trends in Ecology \& Evolution, 22: 148-155.

Bonanomi, S., Pellissier, L., Therkildsen, N. O., Hedeholm, R. B., Retzel, A., Meldrup, D., Olsen, S. M., et al. 2015. Archived DNA reveals fisheries and climate induced collapse of a major fishery. Scientific Reports, 5: 15395.

Butlin, R. K. 2005. Recombination and speciation. Molecular Ecology, 14: 2621-2635.

Cano, J. M., Shikano, T., Kuparinen, A., and Merilä, J. 2008. Genetic differentiation, effective population size and gene flow in marine fishes: implications for stock management. Journal of Integrated Field Science, 5: 1-10.

Carstensen, J., Andersen, J. H., Gustafsson, B. G., and Conley, D. J. 2014. Deoxygenation of the Baltic Sea during the last century. Proceedings of the National Academy of Sciences of the United States of America, 111: 5628-5633.

Dahle, G., Johansen, T., Westgaard, J.-I., Aglen, A., and Glover, K. A. 2018. Genetic management of mixed-stock fisheries "real-time": The case of the largest remaining cod fishery operating in the Atlantic in 2007-2017. Fisheries Research, 205: 77-85.

Darwin, C. R. 1859. On the origin of species by means of natural selection, or the preservation of favoured races in the struggle for life, Murray, London. 502 pp. 
Daverat, F., Morais, P., Dias, E., Babaluk, J., Martin, J., Eon, M., Fablet, R., et al. 2012. Plasticity of European flounder life history patterns discloses alternatives to catadromy. Marine Ecology Progress Series, 465: 267-280.

Erlandsson, J., Östman, Ö., Florin, A.-B., and Pekcan-Hekim, Z. 2017. Spatial structure of body size of European flounder (Platichthys flesus L.) in the Baltic Sea. Fisheries Research, 189: $1-9$.

Fevolden, S., and Pogson, G. 1997. Genetic divergence at the synaptophysin (Syp I) locus among Norwegian coastal and north-east Arctic populations of Atlantic cod. Journal of Fish Biology, 51: 895-908.

Florin, A. B., and Höglund, J. 2008. Population structure of flounder (Platichthys flesus) in the Baltic Sea: differences among demersal and pelagic spawners. Heredity, 101: 27-38.

Friedman, J. H. 1984. A variable span smoother. Laboratory for Computational Statistics, Stanford University Technical Report No. 5.

Funk, W. C., McKay, J. K., Hohenlohe, P. A., and Allendorf, F. W. 2012. Harnessing genomics for delineating conservation units. Trends in Ecology \& Evolution, 27: 489-496.

Gibson, D., Froese, R., Ueberschaer, B., Zylich, K., and Zeller, D. 2015. Flatfishes: Biology and Exploitation, 2nd edn, Wiley \& Sons Ltd, Chichester, UK.

Guo, B., DeFaveri, J., Sotelo, G., Nair, A., and Merilä, J. 2015. Population genomic evidence for adaptive differentiation in Baltic Sea three-spined sticklebacks. BMC Biology, 13: 1-18.

Gustafsson, B. G., and Westman, P. 2002. On the causes for salinity variations in the Baltic Sea during the last 8500 years. Paleoceanography, 17: 12_11-12_14.

Hauser, L., and Carvalho, G. R. 2008. Paradigm shifts in marine fisheries genetics: ugly hypotheses slain by beautiful facts. Fish and Fisheries, 9: 333-362.

Hemmer-Hansen, J., Nielsen, E. E., Grønkjær, P., and Loeschcke, V. 2007. Evolutionary mechanisms shaping the genetic population structure of marine fishes; lessons from the European flounder (Platichthys flesus L.). Molecular Ecology, 16: 3104-3118.

Hemmer-Hansen, J., Nielsen, E. E., Therkildsen, N. O., Taylor, M. I., Ogden, R., Geffen, A. J., Bekkevold, D., et al. 2013. A genomic island linked to ecotype divergence in Atlantic cod. Molecular Ecology, 22: 2653-2667.

Hemmer-Hansen, J., Therkildsen, N. O., and Pujolar, J. M. 2014. Population genomics of marine fishes: next-generation prospects and challenges. The Biological Bulletin, 227: 117-132.

Hensen, V. 1884. Ueber das Vorkommen und die Menge der Eier einiger Ostseefische. Bericht der Commission zur Wissenschaftlichen Untersuchungen der Deutschen Meere, 5: 297-313. 
Hilborn, R., Quinn, T. P., Schindler, D. E., and Rogers, D. E. 2003. Biocomplexity and fisheries sustainability. Proceedings of the National Academy of Sciences of the United States of America, 100: 6564-6568.

Hill, W. G., and Robertson, A. 1968. Linkage disequilibrium in finite populations. Theoretical and Applied Genetics, 38: 226-231.

Hutchinson, W. F. 2008. The dangers of ignoring stock complexity in fishery management: the case of the North Sea cod. Biology Letters, 4: 693-695.

Hyde, J. R., Kimbrell, C. A., Budrick, J. E., Lynn, E. A., and Vetter, R. D. 2008. Cryptic speciation in the vermilion rockfish (Sebastes miniatus) and the role of bathymetry in the speciation process. Molecular Ecology, 17: 1122-1136.

ICES. 2017. ICES WGBFAS REPORT 2016: Report of the Baltic Fisheries Assessment Working Group (WGBFAS). ICES Document ICES CM 2016/ACOM:11. 795 pp.

Jokinen, H., Wennhage, H., Lappalainen, A., Ådjers, K., Rask, M., and Norkko, A. 2015. Decline of flounder (Platichthys flesus (L.)) at the margin of the species' distribution range. Journal of Sea Research, 105: 1-9.

Kijewska, A., Kalamarz-Kubiak, H., Arciszewski, B., Guellard, T., Petereit, C., and Wenne, R. 2016. Adaptation to salinity in Atlantic cod from different regions of the Baltic Sea. Journal of Experimental Marine Biology and Ecology, 478: 62-67.

Knowlton, N. 2000. Molecular genetic analyses of species boundaries in the sea. In Marine genetics, pp. 73-90. Ed. by A. M. Solé-Cava, C. A. M. Russo, and J. P. Thorpe. Springer.

Lamichhaney, S., Barrio, A. M., Rafati, N., Sundström, G., Rubin, C.-J., Gilbert, E. R., Berglund, J., et al. 2012. Population-scale sequencing reveals genetic differentiation due to local adaptation in Atlantic herring. Proceedings of the National Academy of Sciences of the United States of America, 109: 19345-19350.

Lønning, S., and Solemdal, P. 1972. The relation between thickness of chorion and specific gravity of eggs from Norwegian and Baltic flatfish populations. Fiskeridirektoratets skrifter, Serie Havundersøkelse, 16.

Manel, S., Schwartz, M. K., Luikart, G., and Taberlet, P. 2003. Landscape genetics: combining landscape ecology and population genetics. Trends in Ecology \& Evolution, 18: 189-197.

Matsubara, T., Ohkubo, N., Andoh, T., Sullivan, C. V., and Hara, A. 1999. Two forms of vitellogenin, yielding two distinct lipovitellins, play different roles during oocyte maturation and early development of barfin flounder, Verasper moseri, a marine teleost that spawns pelagic eggs. Developmental Biology, 213: 18-32. 
Momigliano, P., Jokinen, H., Fraimout, A., Florin, A.-B., Norkko, A., and Merilä, J. 2017.

Extraordinarily rapid speciation in a marine fish. Proceedings of the National Academy of Sciences of the United States of America, 114: 6074-6079.

Momigliano, P., Denys, G. P., Jokinen, H., and Merilä, J. 2018. Platichthys solemdali sp. nov.(Actinopterygii, Pleuronectiformes): a new flounder species from the Baltic Sea. Frontiers in Marine Science, 5: 225.

Momigliano, P., Jokinen, H., Calboli, F., Aro, E., and Merilä, J. 2019. Cryptic temporal changes in stock composition explain the decline of a flounder (Platichthys spp.) assemblage. Evolutionary Applications, 12: 549-559.

Nielsen, E. E., Hemmer-Hansen, J., Larsen, P. F., and Bekkevold, D. 2009. Population genomics of marine fishes: identifying adaptive variation in space and time. Molecular Ecology, 18: 3128-3150.

Nissling, A., Westin, L., and Hjerne, O. 2002. Reproductive success in relation to salinity for three flatfish species, dab (Limanda limanda), plaice (Pleuronectes platessa), and flounder (Pleuronectes flesus), in the brackish water Baltic Sea. ICES Journal of Marine Science, 59: 93-108.

Nissling, A., and Dahlman, G. 2010. Fecundity of flounder, Pleuronectes flesus, in the Baltic SeaReproductive strategies in two sympatric populations. Journal of Sea Research, 64: 190-198. Nissling, A., Nyberg, S., and Petereit, C. 2017. Egg buoyancy of flounder, Platichthys flesus, in the Baltic Sea - adaptation to salinity and implications for egg survival. Fisheries Research, 191: 179-189.

Nissling, A., and Larsson, R. 2018. Population specific sperm production in European flounder Platichthys flesus: Adaptation to salinity at spawning. Journal of Fish Biology, 93: 47-52.

Nosil, P. 2012. Ecological speciation, Oxford University Press.

Ortiz-Barrientos, D., Engelstädter, J., and Rieseberg, L. H. 2016. Recombination rate evolution and the origin of species. Trends in Ecology \& Evolution, 31: 226-236.

Puebla, O. 2009. Ecological speciation in marine v. freshwater fishes. Journal of Fish Biology, 75: 960-996.

Reiss, H., Hoarau, G., Dickey-Collas, M., and Wolff, W. J. 2009. Genetic population structure of marine fish: mismatch between biological and fisheries management units. Fish and Fisheries, 10: 361-395.

Rocha, L. A., Robertson, D. R., Roman, J., and Bowen, B. W. 2005. Ecological speciation in tropical reef fishes. Proceedings of the Royal Society B: Biological Sciences, 272: 573-579. 
Savolainen, O., Lascoux, M., and Merila, J. 2013. Ecological genomics of local adaptation. Nature Reviews: Genetics, 14: 807-820.

Schluter, D., and Rambaut, A. 1996. Ecological speciation in postglacial fishes [and discussion]. Philosophical Transactions of the Royal Society of London, Series B: Biological Sciences, 351: 807-814.

Schluter, D., and Conte, G. L. 2009. Genetics and ecological speciation. Proceedings of the National Academy of Sciences of the United States of America, 106: 9955-9962.

Selkoe, K. A., Aloia, C. C., Crandall, E. D., Iacchei, M., Liggins, L., Puritz, J. B., von der Heyden, S., et al. 2016. A decade of seascape genetics: contributions to basic and applied marine connectivity. Marine Ecology Progress Series, 554: 1-19.

Servedio, M. R., Van Doorn, G. S., Kopp, M., Frame, A. M., and Nosil, P. 2011. Magic traits in speciation:'magic'but not rare? Trends in Ecology \& Evolution, 26: 389-397.

Solemdal, P. 1967. The effect of salinity on buoyancy, size and development of flounder eggs. Sarsia, 29: 431-442.

Solemdal, P. 1973. Transfer of Baltic flatfish to a marine environment and the long termeffects on reproduction. Oikos, (suppl.) 15: 268-276.

Turner, R. M. 2003. Tales from the tail: what do we really know about sperm motility? Journal of andrology, 24: 790-803.

Vuorinen, I., Hänninen, J., Rajasilta, M., Laine, P., Eklund, J., Montesino-Pouzols, F., Corona, F., et al. 2015. Scenario simulations of future salinity and ecological consequences in the Baltic Sea and adjacent North Sea areas-implications for environmental monitoring. Ecological Indicators, 50: 196-205.

Ye, X., Chun, J., and Metabolism 2010. Lysophosphatidic acid (LPA) signaling in vertebrate reproduction. Trends in Endocrinology, 21: 17-24.

Zuccarello, D., Ferlin, A., Cazzadore, C., Pepe, A., Garolla, A., Moretti, A., Cordeschi, G., et al. 2008. Mutations in dynein genes in patients affected by isolated non-syndromic asthenozoospermia. Human reproduction, 23: 1957-1962. 
Figure legends
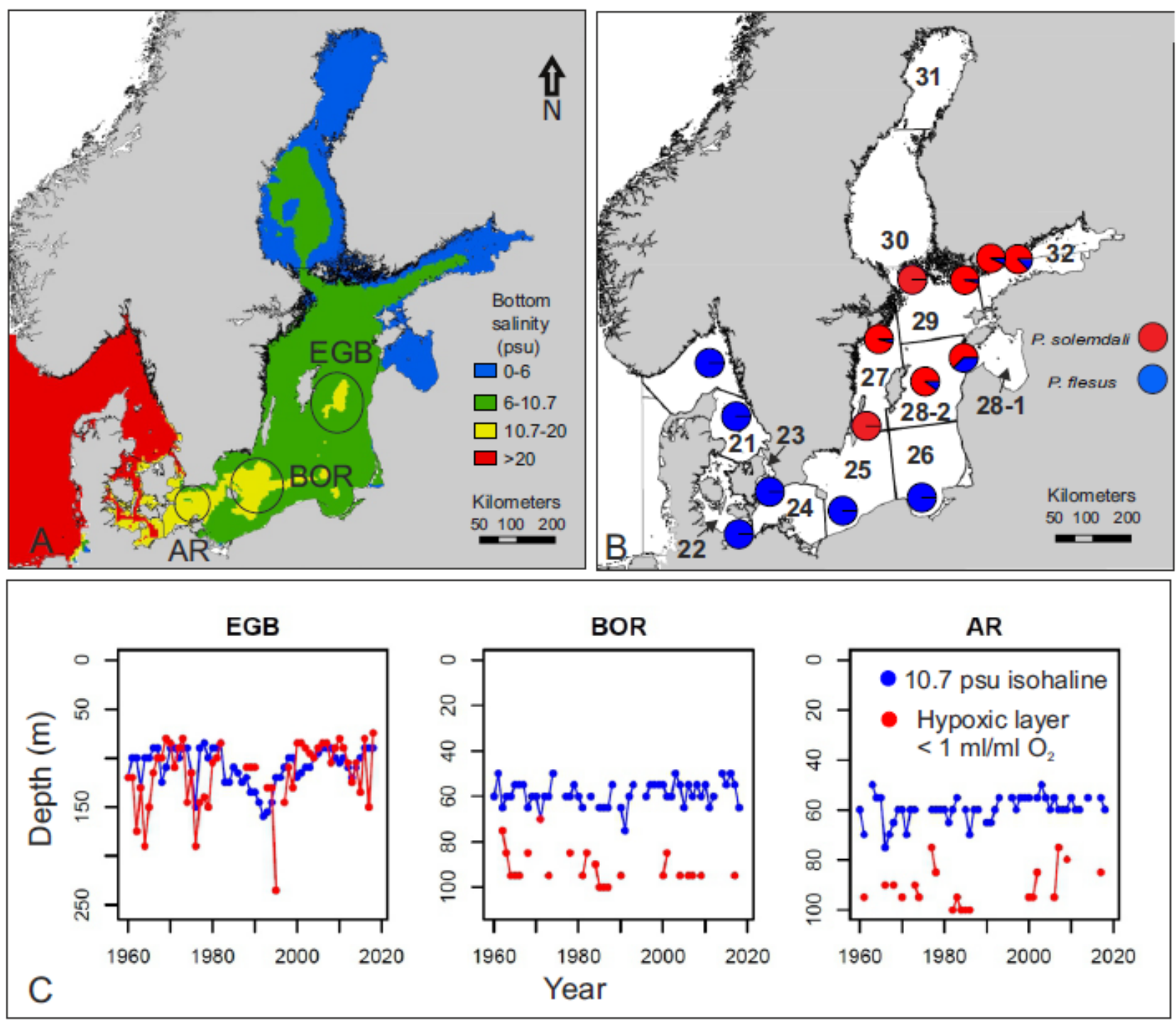

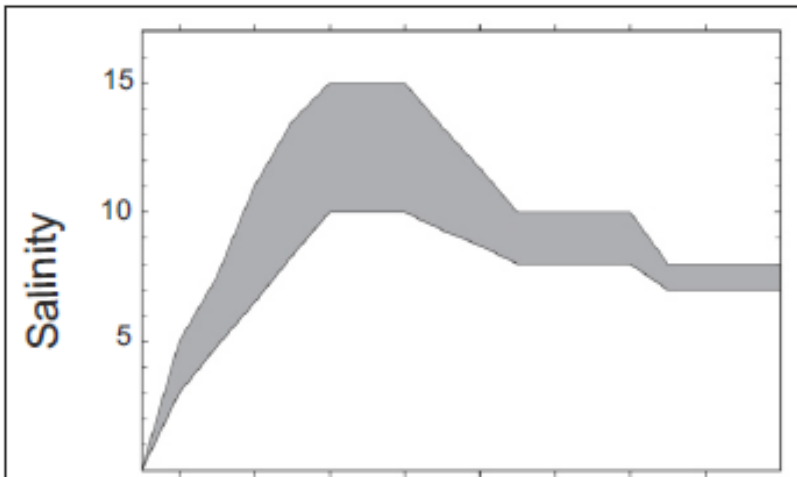

80007000600050004000300020001000

D Calendar years BP

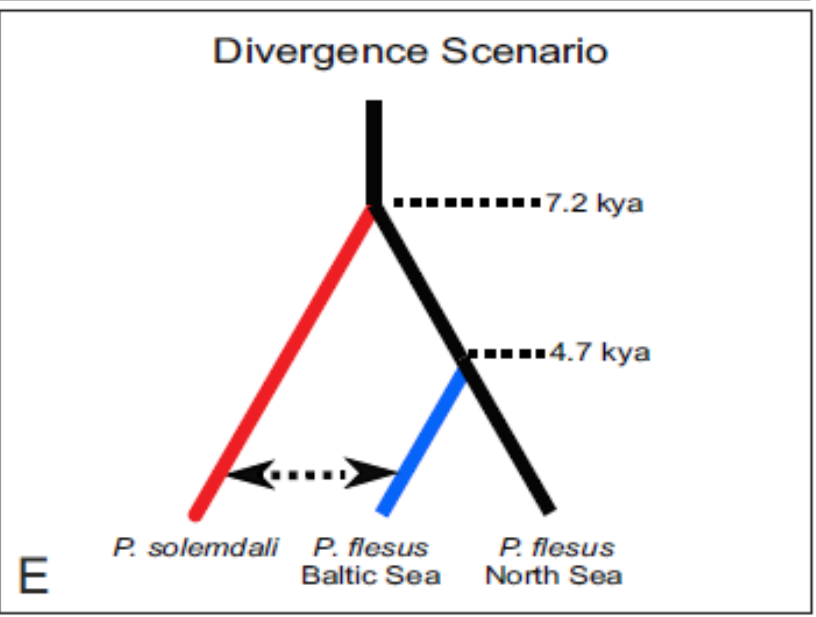


Fig. 1 A Map of the Baltic Sea showing modelled mean bottom salinity from Bendtsen et al. (2007), showing the location of the main three spawning grounds of $P$. flesus: the Arkona basin (AR), the Bornholm Basin (BOR) and the Eastern Gotland Basin (EGB). Areas in and yellow and red have salinities at which $P$. flesus can spawn (>10.7 psu). Areas in green have bottom salinities above 6 psu (the salinity at which $P$. solemdali can reproduce successfully). B: contemporary proportion of the three main flounder lineages (North Sea P. flesus, Baltic Sea P. flesus and P. solemdali) in different localities and across ICES subdivisions of the Baltic Sea (SD 21-32), based on previously published data (Momigliano et al., 2017; 2018; 2019). It should be noted that these do not represent accurate and stable proportion in each of the localities, as they are likely affected by sampling time (both within and between year), but they do provide evidence of mixed stock in several locations. $\mathrm{C}$ : temporal changes in the $10.7 \mathrm{psu}$ isohaline, and in the depth of the hypoxic layer (dissolved $\mathrm{O} 2$ $<1 \mathrm{mg} / \mathrm{ml}$ ), in the AR, BOR and EGB. When the hypoxic layer is below the $10.7 \mathrm{psu}$ isohaline $P$. flesus reproduction is possible. D: estimated of the mean salinity of the Baltic Sea during the Littorina Sea stage based on proxy data from Gustafsson and Westman (2002). E: divergence scenario between the three flounder lineages from Momigliano et al. (2017), showing an initial allopatric phase between $P$. flesus and the ancestor of $P$. solemdali and introgression following the second invasion of the Baltic Sea by P. flesus. 


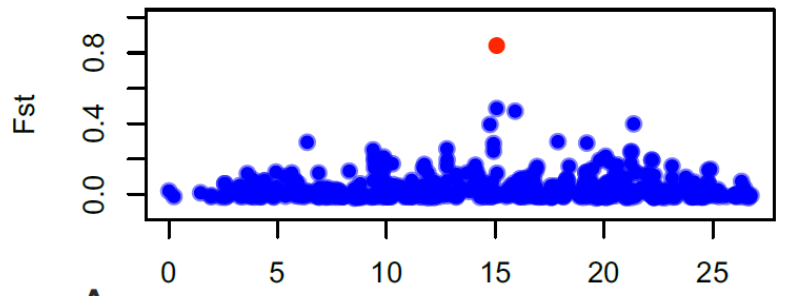

Position (Mb, Scaffold 1)

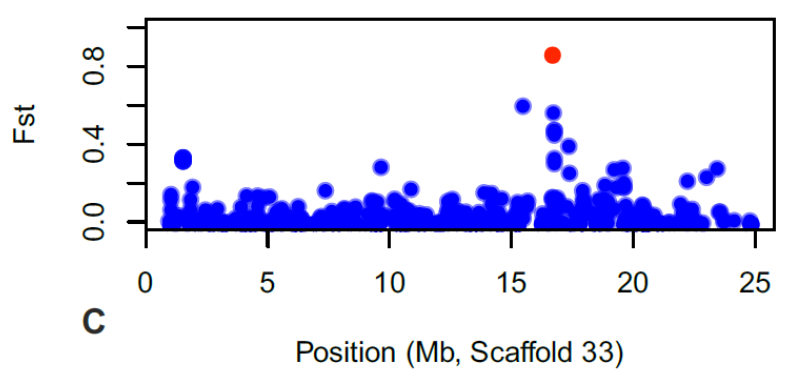

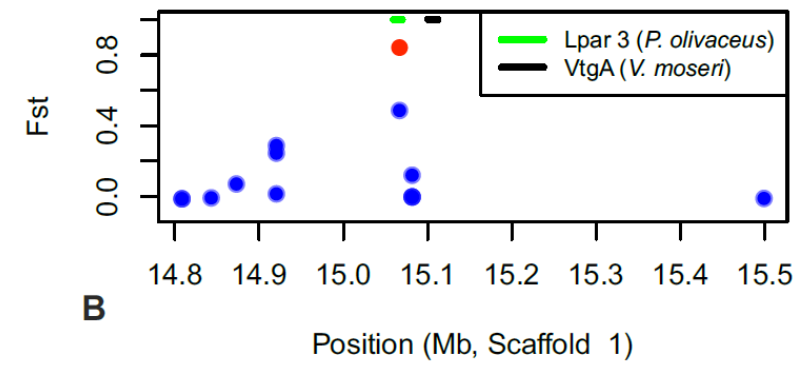

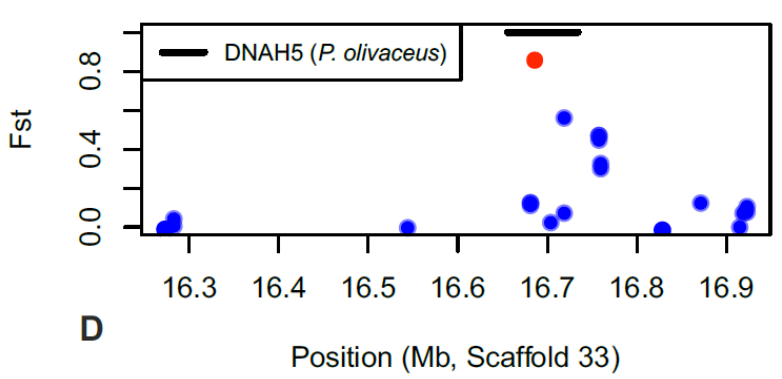

Fig. 2 Genetic differentiation $\left(F_{\mathrm{ST}}\right)$ between P. solemdali and P. flesus along the two scaffolds where the divergent outlier loci 886_19 and 3599_4 (Momigliano et al., 2017a) are located. The red symbols indicate outlier 886_19 (A, B) and 3599_4 (C, D). On the left panel are $F_{\text {ST }}$ across the scaffold (A, C), after the raw reads from Momigliano et al. (2017) were realigned along the draft genome. On the right graphs $(C, D)$ the specific regions of the scaffold where outliers were found are superimposed. On the top of graphs C \& D the lines represent the regions to which candidate gene matches from the closely related species map (Lpar 3 from P. olivaceus and VtgA from outlier 886_19, and DNAH5 from P. olivaceus for $\left.3599 \_4\right)$. 

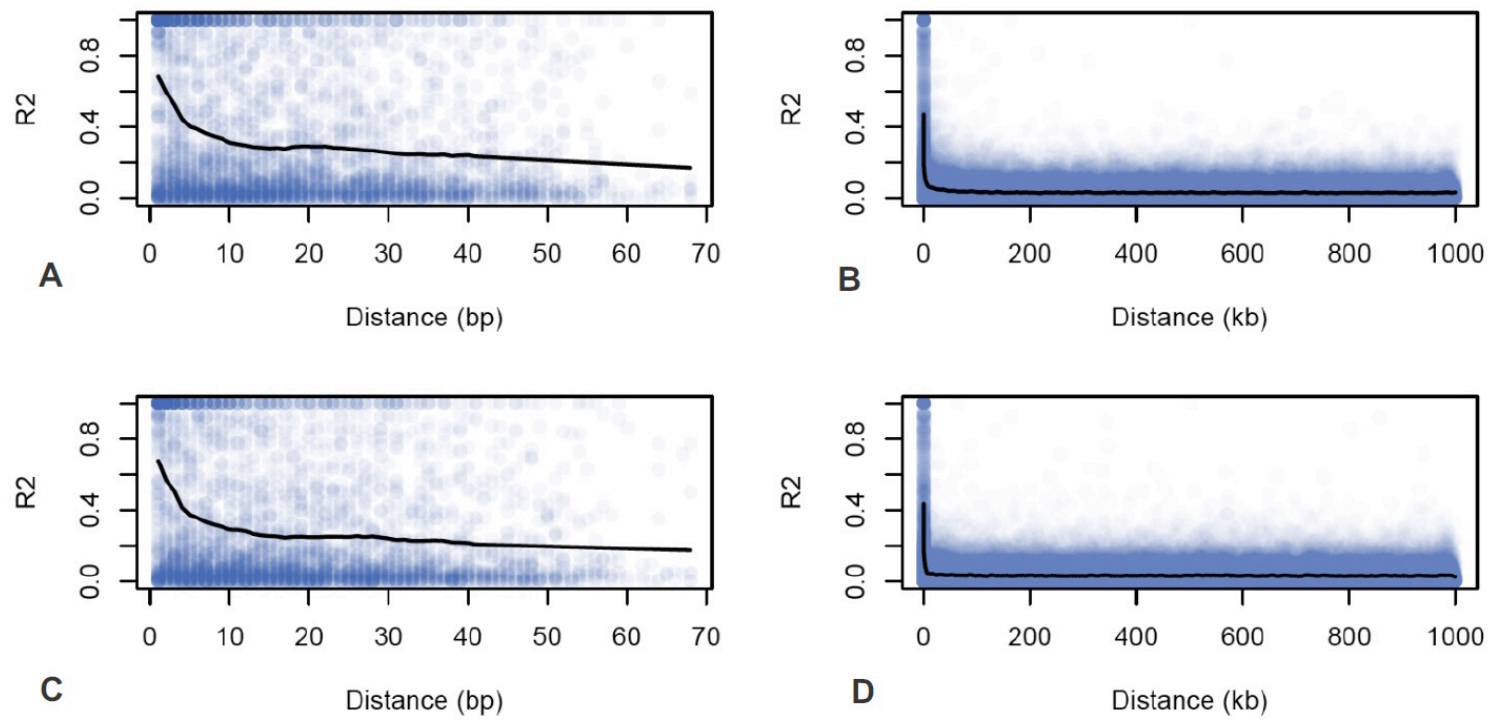

Fig. 3 Decay of linkage equilibrium in P. solemdali (A, B) and P. flesus (C, D). Graphs A and C show how LD between pairs of SNPs decays rapidly even within single DArT loci (69 bp in length). Graphs B and D show LD calculate across the draft genome for pairs of SNPs within $1 \mathrm{mb}$ of distance. LD drops to background level within just over $1000 \mathrm{bp}$. Transparency is used to show the density of points and black lines represent Friedman's super smoother (Friedman, 1984).

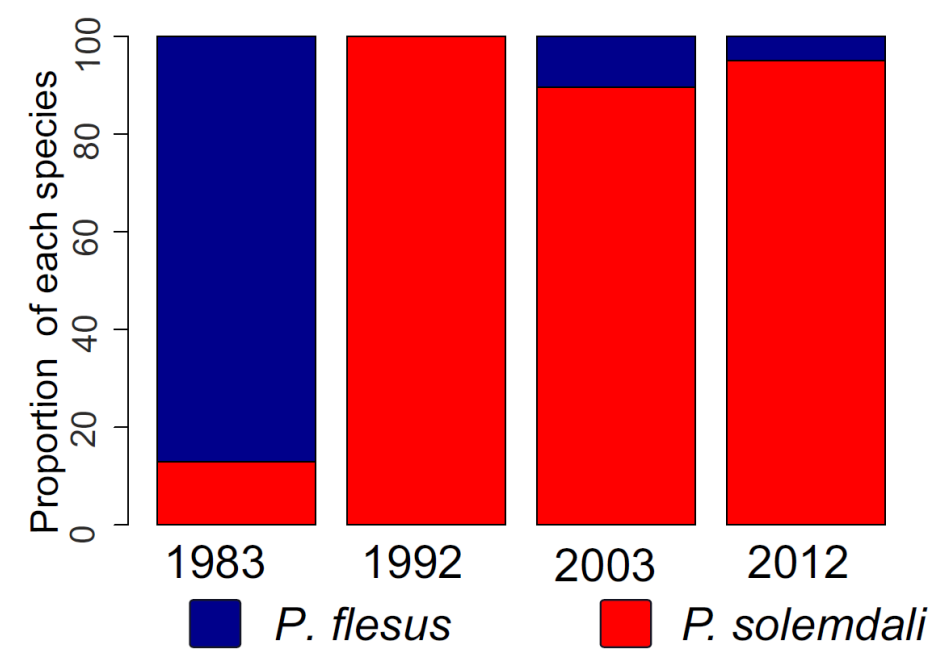

Fig. 4 Temporal changes in the relative proportion of P. flesus and P. solemdali in the Gulf of Finland (Momigliano et al., 2019). 